\title{
Metanephric Tumor
}

National Cancer Institute

\section{Source}

National Cancer Institute. Metanephric Tumor. NCI Thesaurus. Code C157748.

A neoplasm arising from the distal convoluted tubule and collecting duct areas of the kidney. 